\title{
A!
}

This is an electronic reprint of the original article.

This reprint may differ from the original in pagination and typographic detail.

Mahboob Kanafi, Mona; Tuononen, Ari J.

\section{Top topography surface roughness power spectrum for pavement friction evaluation}

Published in:

Tribology International

DOI:

10.1016/j.triboint.2016.11.038

Published: 01/03/2017

Document Version

Peer reviewed version

Published under the following license:

CC BY-NC-ND

Please cite the original version:

Mahboob Kanafi, M., \& Tuononen, A. J. (2017). Top topography surface roughness power spectrum for pavement friction evaluation. Tribology International, 107, 240-249. https://doi.org/10.1016/j.triboint.2016.11.038

This material is protected by copyright and other intellectual property rights, and duplication or sale of all or part of any of the repository collections is not permitted, except that material may be duplicated by you for your research use or educational purposes in electronic or print form. You must obtain permission for any other use. Electronic or print copies may not be offered, whether for sale or otherwise to anyone who is not an authorised user. 


\title{
Top Topography Surface Roughness Power Spectrum for Pavement Friction Evaluation
}

\author{
Mona Mahboob Kanafi ${ }^{1 *}$, Ari Juhani Tuononen ${ }^{1}$ \\ Vehicle Engineering Group, School of Engineering, Aalto University, P.O. Box 14300, FI-00076 Aalto, Helsinki, Finland \\ * mona.mahboobkanafi@aalto.fi
}




\begin{abstract}
This article deals with the most suitable calculation procedure for top topography surface roughness power spectrum (PSD). The information top PSD provides about the roughness characteristics and its practical application in tyre-road friction studies are covered. The influence of portions of top topography used for calculations on the realization these PSDs give about surface height distributions is investigated. Results of roughness PSDs generally proved to be dependent on portions of top topography used for calculations. A high correlation with an average around 0.8 was found with friction and top $20 \%$ of PSDs, but only at a short-scale surface roughness $\lambda \leq 1 \mathrm{~mm}$. Low correlation coefficients between friction and longer $\lambda$ were discussed through the depth of the penetration of the rubber into each pavement.
\end{abstract}

\title{
Keywords
}

Tire/Road; Friction; Surface Roughness; Power Spectrum; 


\section{Introduction}

Rubber friction has long attracted the interest of many physicists, tyre experts and pavement engineers. With the recent development of the theories of rubber friction and contact mechanics [1-7], the complexity of the relation between road surface roughness and rubber friction is now more highlighted than before. Although many experimental works have addressed this connection [8-20], the multiscale nature of the pavement surface roughness and the partial contact of the tyre rubber with this roughness profile make it difficult to explore meaningful relations between friction and simple roughness indicators.

When dealing with this, the first step is to introduce roughness indicators that can fully characterize the top surface topography (tyre-road contact zone) on different length scales. Persson [2] employed the surface roughness power spectrum (PSD) for the characterization of randomly rough surfaces in multiscale and developed a theory of rubber friction for the sliding of a rubber block on a rough surface, with roughness on many different length scales. Chen and Wang [8], applying PSD to study the evolution of the surface profile of aggregates in the laboratory polishing process, reported changes in the micro-roughness as the main contribution to the loss of friction. However, Mahboob Kanafi et al. [15], employing the same roughness characterization method in a field experiment, concluded that the macro/micro-roughness variations of road pavements occur within the full surface topography and not exclusively on the top profile; thus, observing a pattern between full-profile PSD variations and the evolution of friction still remained a challenge in actual field conditions. In the field experiment of Hartikainen et al. [13], frequency-wise correlation of pavement friction with the PSD calculated only on the top profile (a technique first introduced by Persson et al. [21]) was conducted, with the highest correlation being found at the longest length scale under investigation.

Although so far the top power spectrum appears to be the best technique to characterize the road surface profiles for tyre-road studies, the knowledge of the approperiate calculation of the top PSD and how the resulting top PSD must be interpreted in the calculations of the contact mechanics is still incomplete. In this regard, here, we dedicate the next section to a review of the characterization of the top surface topography of pavements by presenting the most suitable calculation procedure for the top PSD and illustrating the actual information it gives about the height distribution of a surface that is obtained through this top PSD. The application of this technique in the field experiments is then investigated thoroughly, using experimental data given in Section 3, inclusive of the monitoring of four road pavements during a nine-month evaluation period. In Section 4, a discussion is presented on the depth/portion of the top topography that is relevant for the top PSD calculations during tyre-road sliding contact and a rough estimate of this portion is then given for the experimental data. This is followed by sections providing the results and conclusions concerning the correlation between friction and the top PSD at each of the length scales under study, where the influence of the portion of the surface topography used in the top PSD calculations is also explored on the correlation results. 


\section{Top topography power spectrum}

For a randomly rough surface, when the surface height distribution can be approximated with a Gaussian distribution, all the statistical properties of the surface roughness are contained in the surface roughness power spectrum $\mathrm{C}(\mathrm{q})$ where $\mathrm{q}$ is the roughness wavevector [21]. However, the surface characterization of asphalt pavements has, in many cases, shown non-random distribution of heights $[13,15]$. This skewed texture could arise from the hot mix asphalt compaction process or pavement mix types such as open-graded mixes, etc., but the magnitude of the pavement skewness also evolves through the year as a result of the change to warm seasons from cold periods and vice versa [15]. For a skewed pavement surface, any indicator of the surface roughness derived from the full surface profile overshadows the true roughness that actually contributes to the tyre/road contact. The conditions under which the tyre rubber is able to deform and make contact with the pavement surface everywhere (full contact) depend on the perpendicular pressure $\sigma_{0}$. At the least, a local pressure in the order of the elastic modulus of the filled rubber $\mathrm{E} \approx 10$ MPa (static case) is needed to satisfy the latter condition [2]. Thus, only partial contact is normally expected for typical passenger/truck tyre applications $\left(\sigma_{0}<1 \mathrm{MPa}\right)$, which only occur on top of the highest asperities. While pavement skewness escalates roughness indicators such as MPD (mean profile depth), $R_{q}$ (root mean square roughness) and even the energy represented at different frequencies of the roughness PSD, the increase in the parameters has no influence on the area of real contact, leading to false conclusions when these indicators are correlated with friction data. For this reason, there is an urge towards the characterization of surface roughness only on the top topography of pavements, still representing roughness at different length scales.

Ueckermann et al. [17] used summit profile PSD in order to exclude the surface cavities of the pavement. However, as also mentioned by the same authors, this method alters the information on the low-frequency components of the surface roughness. Altering texture components is not accepted in this context as all roughness wavelengths are a priori equally important in contact mechanics [2]. The top and bottom power spectrum approach was first introduced by Persson et al. [21] for surface characterization, defined by:

$$
\begin{aligned}
& C_{T}(q)=\frac{1}{(2 \pi)^{2}} \int d^{2} x\left\langle h_{T}(x) h_{T}(0)\right\rangle e^{-i q \cdot x} \\
& C_{B}(q)=\frac{1}{(2 \pi)^{2}} \int d^{2} x\left\langle h_{B}(x) h_{B}(0)\right\rangle e^{-i q \cdot x}
\end{aligned}
$$

where $h_{T}(x)=h(x)$ for $h>0$ and zero otherwise, $h_{B}(x)=h(x)$ for $h<0$ and zero otherwise, and $\langle\ldots\rangle$ stands for ensemble averaging, i.e. averaging over a collection of surfaces with the same statistical properties [21]. Although this approach was first presented by Persson et al. [21], the concept of its calculation and the realization it gives about the surface roughness components of a pavement require additional explanation. In general, the top power spectrum could be calculated for any portion of the top surface topography and not just above/below the average plane. In the work of Hartikainen et al. [13], the frequncy-wise correlation of the top power spectrum (calculated at a specific depth of height profile) with friction was studied for the first time; however, the concept of the calculation procedure is somehow not well identified. Here, we demonstrate that what Hartikainen et al. [13] refer to as the "areacorrected/non-corrected top power spectrum" is only related to assigning the correct root mean square roughness $R_{q}$ to the calculated top power spectrum. Hence, the area correction or what we call here the $\mathrm{R}_{\mathrm{q}}$ correction must be applied for an approperiate calculation of the top power spectrum, as described below.

The Fourier transform is a linear transform:

$\mathrm{F}\left\{h_{T}(x)+h_{B}(x)\right\}=\mathrm{F}\left\{h_{T}(x)\right\}+\mathrm{F}\left\{h_{B}(x)\right\}$

$C(q)=C_{T}(q)+C_{B}(q)$

where $\mathrm{F}$ denotes the Fourier transform and $\mathrm{C}(\mathrm{q})$ refers to the full surface roughness power spectrum. As a result, the top and bottom halves of a surface profile must have lower power than the full topography. For illustrative purposes, we consider a randomly rough fractal surface with the full power spectrum presented in Fig. 1 (a). We know that the 
top and bottom surface roughness power spectra of a fractal surface must have the same scaling as well as surface roughness energy at different frequencies than that of the full power spectrum, which is not the case for the calculations presented above (Fig. 1(a), top and bottom profile, each occupying 50\% of the projected surface area). As stated in [21], if $n_{T}$ and $n_{B}$ are the fraction of the surface area where $h>0$ and $h<0$, respectively, with the condition that $n_{T}+n_{B}=1$, i.e. :

$n_{T}=\frac{N_{T}}{N}$

$n_{B}=\frac{N_{B}}{N}$

where $N=N_{T}+N_{B}$ is the total number of data points in the full surface profile, and $\mathrm{N}_{\mathrm{T}}$ and $\mathrm{N}_{\mathrm{B}}$ are the corresponding number of data points in the top and bottom topography. We have [21]:

$$
\begin{gathered}
C^{*}{ }_{T}=\frac{C_{T}}{n_{T}} \\
C^{*}{ }_{B}=\frac{C_{B}}{n_{B}}
\end{gathered}
$$

which we call the $R_{q}$ corrected top and bottom power spectra of surface roughness (Fig. 1(b)). The reason behind this correction is the true energy (root mean square roughness) represented in the power spectral density graph. By definition, the root mean square roughness of a profile is:

$R_{q}^{2}=\frac{1}{N} \sum_{n=1}^{N}|h(x)|^{2}$

However, when we calculate the top power spectrum $C_{T}(q)$, the $R_{q}$ that is actually represented in the top PSD is lower than the actual $R_{q}$ of the top topography as a result of the fact that the bottom profile is replaced with zero components:

Calculated $R_{q}{ }^{2}=\frac{1}{N} \sum_{1}^{N}\left|h_{T}(x)\right|^{2}$

Actual $R_{q}{ }^{2}=\frac{1}{N_{T}} \sum_{1}^{N}\left|h_{T}(x)\right|^{2}$

In terms of conservation of energy (Parseval's theorem), the area under the power spectral density curve equals $R_{q}{ }^{2}$ :

$R_{q}^{2}=\frac{(2 \pi)^{2}}{A} \sum_{1}^{n} \sum_{1}^{m}|C(q)|^{2}$

where $\mathrm{A}$ is the surface area under study, and $\mathrm{n}$ and $\mathrm{m}$ are the grid of measured pixels in the surface height map. Now, in order for the calculated top power spectrum in Eq. (1) to have the actual $R_{q}$ presented in Eq. (11), one needs to divide the top power spectrum by $n_{T}$, which will result in an $R_{q}$-corrected top power spectrum $C^{*}{ }_{T}$ in Eq. 7 . The same is true for the bottom power spectrum. On this account, it is necessary to compensate for the reduction in the $R_{q}$ when calculating the top/bottom power spectrum of a surface topography. 

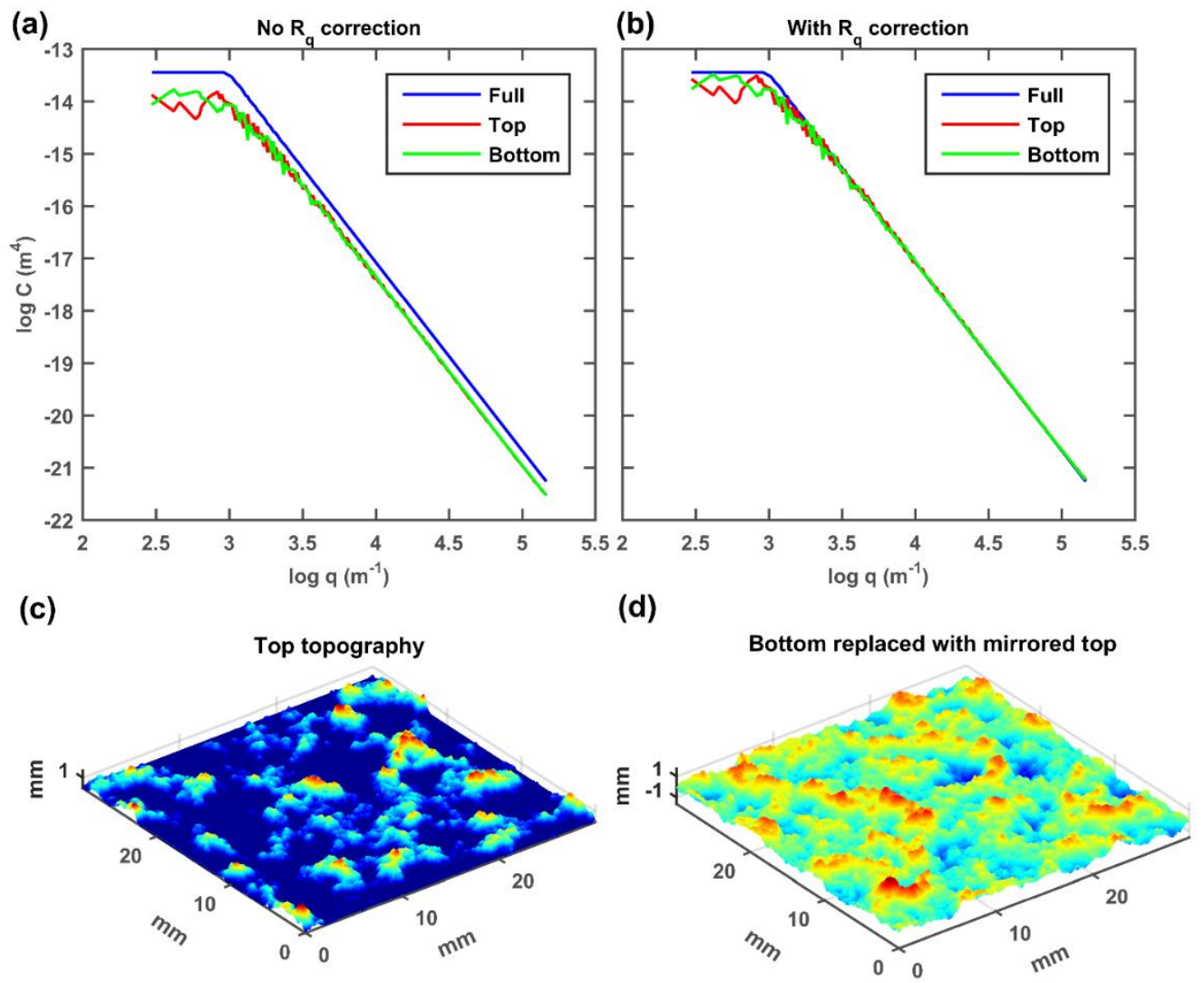

Fig. 1. Top topography analysis of a fractal surface. (a): Full, top and bottom power spectra with no correction of $R_{q}$. (b) Compensating for the reduction in $R_{q}$ for top and bottom power spectrum. (c): Top topography of a fractal surface. (d): Generated artificial surface from the top topography PSD presented in (b) but with random phase, and with exactly the same statistical properties as the top topography in (c).

Here, it is better to explain the information that the $\mathrm{R}_{\mathrm{q}}$-corrected top PSD brings about the surface roughness characteristics. We generate an artificial surface via the top PSD presented in Fig. 1(b) that was already calculated from the top 50\% $\left(n_{T}=n_{B}=0.5\right)$ topography in Fig. 1(c), using a random phase in the range [0 $\left.2 \pi\right]$. This results in a surface topography (Fig. 1(d)) which Persson et al. [21] described as the bottom profile being replaced by a mirrored top profile. In reality, even though the generated top surface is not exactly the same as the original top topography, it gives the same perception about the statistical roughness properties of the original top profile. Eventually, for a randomly rough surface, the phase information in the calculated PSD which describes all the positioning information about the surface roughness is unimportant (concept of randomness); thus, the $\mathrm{R}_{\mathrm{q}}$-corrected top PSD (from now on we call it the top PSD) could completely characterize the surface roughness characteristics of a surface profile of a random nature.

The issue dealt with above considers the top PSD calculated from a top cut of the surface topography at $50 \%$ surface area. Now, we discuss what the cut-topography at different depths of the surface profile says about the surface roughness characteristics and asperity distribution on the top topography. If we cut a fractal surface at different heights above the average plane (mean, median and mode of the height distribution at $h=0$ ) as illustrated in Fig. 2(a), we can see that the calculated top PSDs in Fig. 2(b) reveal the true scaling of the short-scale roughness components. It is evident that the long-scale components of the surface roughness are filtered out when we choose smaller portions of the top topography. In the context of tyre-road contact, this does not mean altering long-wavelength components, as is the case with summit profile analysis [17]. The filtering implies that if the depth of the penetration of the rubber 
into the road surface asperities is at this height level, in practice the rubber cannot see the longer wavelengths that exist beyond this level.
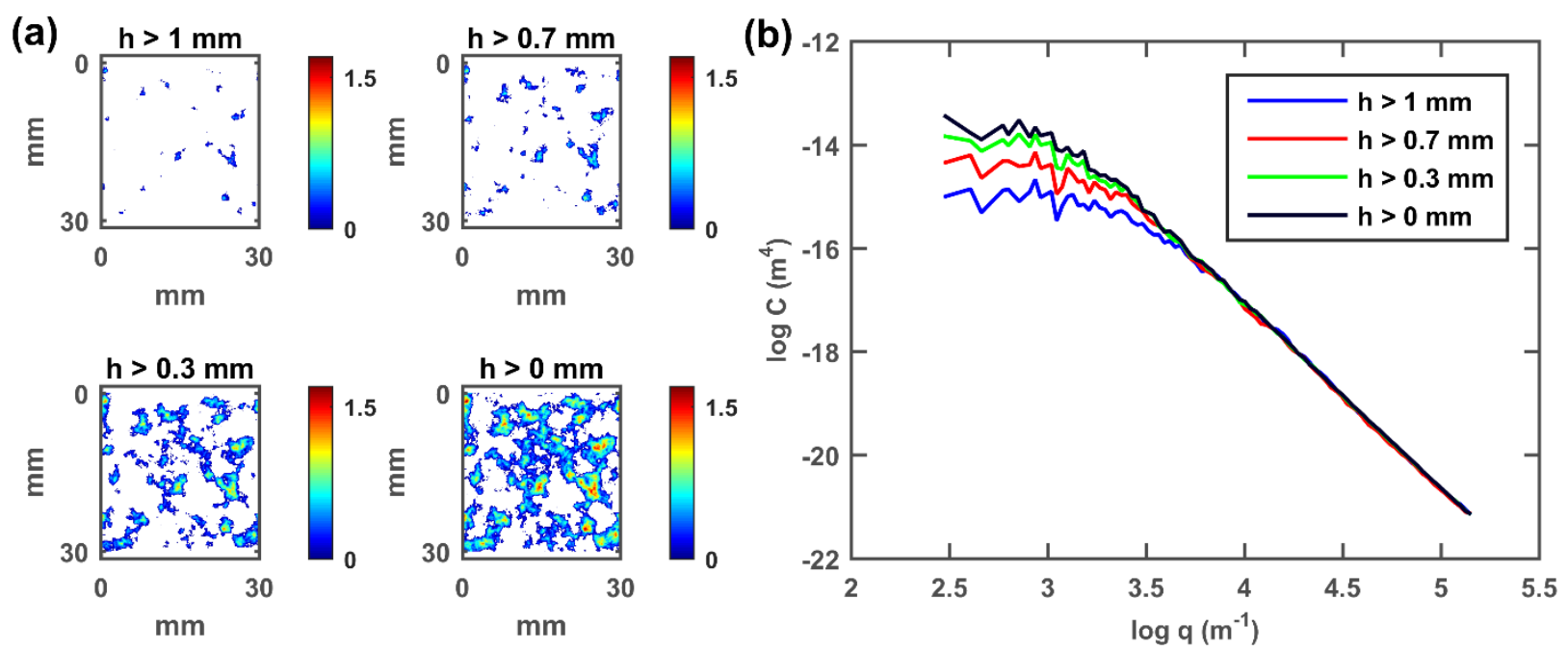

Fig. 2. On the effect of cut depth on surface roughness power spectrum. (a): Surface top topography of a fractal surface at different cut depths. (b): Effect of cut depths on top PSD.

However, even though the correct scaling and short-scale roughness components are well preserved in the calculated top power spectrum, the calculations of the contact mechanics using any top power spectrum other than the top 50\% lead to false conclusions. Looking at Fig. 3(a) and (c), the two topographies shown share the same surface roughness power spectrum as that given in Fig. 3(b), but have totally different asperity distributions. In other words, the distribution of the macroasperity contact regions will be completely different for the two illustrated surfaces. This arises from the percentage of the data and the corresponding $R_{q}$ for that portion of the surface. For instance, considering the top 20\% topography in Fig. 3(a), the $\mathrm{R}_{\mathrm{q}}$ of this portion of the surface is the same as the $\mathrm{R}_{\mathrm{q}}$ of the full topography of the generated surface in Fig. 3(c) (likewise, the top and bottom $50 \%$ of the data have almost the same $\mathrm{R}_{\mathrm{q}}$ ). It is worth mentioning that only the top half of the topography is shown in Fig. 3(c) with a view to better illustrating the distribution of the asperities. Thus, if only the top 50\% of the data is chosen for analysis, the contact configuration will be fully characterized by means of the top power spectrum, as holds for Fig. 1(c) and (d). This is an important conclusion about the range of applicability of the top PSD method for the calculation of the contact mechanics.

(a)

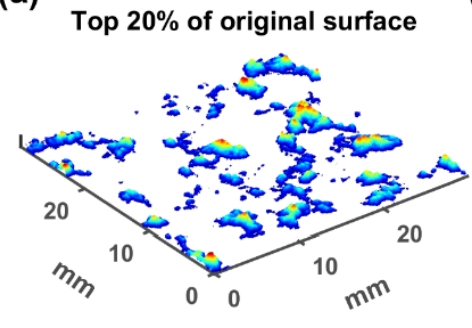

(b)

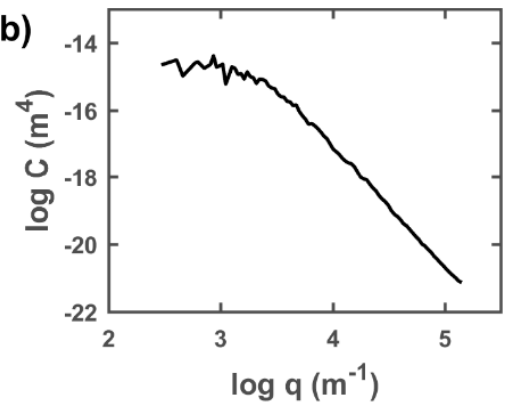

(c)

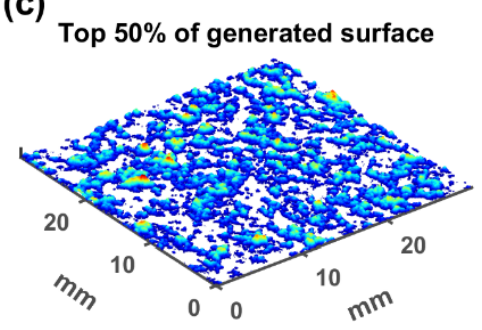

Fig. 3. Two topographies sharing the same PSD but with different asperity distribution. (a): Top $20 \%$ of a fractal surface. (b): Calculated top PSD for case (a). (c): Surface generated again from the PSD presented in (b); only the top 50\% is shown for illustrative purposes. 


\section{Experimental data}

The experimental data presented here was obtained during the monitoring of four pavements (Table 1) in Finland. Friction and 3D surface topography measurements of the pavements were conducted during a short period of nine months, from March to November, and only in dry surface conditions. Throughout this period, seven topography/friction data sets were obtained from the wearing course of the pavements in a 4-m evaluation length. Each data set was acquired during a two-hour time span; therefore, the air temperature in which the friction data was collected was almost the same for the four pavements. The air temperature was obtained from the observation station of the Finnish Meteorological Institute. The friction tester used was portable ASFT T2GO (ASFT Industries AB ${ }^{\mathrm{TM}}$, Sweden) equipment with a fixed slip ratio of $20 \%$. All the friction measurements were carried out under dry surface conditions. The measurements of the surface roughness were carried out by means of an optical portable non-contact 3D-profilometer (GFM MikroCAD, Germany). At least 10 images were captured for a single pavement, each image being $46 \times 29 \mathrm{~mm}^{2}$ and with a height and lateral resolution of $3 \mu \mathrm{m}$ and $61 \mu \mathrm{m}$, respectively. A more detailed explanation of the experimental procedure can be found in [15].

Table 1

Specification of roads studied.

\begin{tabular}{llllllll}
\hline Site & Road type & $\begin{array}{l}\text { Speed limit } \\
(\mathrm{km} / \mathrm{h})\end{array}$ & $\begin{array}{l}\text { Pavement age } \\
\text { (years) }\end{array}$ & Pavement type & $\begin{array}{l}\text { AADT } \\
\text { (vehicles/day) }\end{array}$ & $\begin{array}{l}\text { Heavy } \\
(\%)\end{array}$ & vehicles $^{\mathrm{a}}$ \\
\hline $\mathrm{A}^{\mathrm{b}}$ & Access road & 40 & $>10$ & AB16 & $<300$ & $<0.5$ & \\
$\mathrm{~B}^{\mathrm{b}}$ & Collector road & 50 & 3 & SMA11 & 10,000 & 10.7 & \\
$\mathrm{C}^{\mathrm{b}}$ & Collector road & 40 & 3 & SMA11 & 12,000 & 4.5 & \\
$\mathrm{D}^{\mathrm{b}}$ & Ring road & 70 & 3 & SMA16 & 45,300 & 7 & \\
\hline
\end{tabular}

Note: AADT, Annual Average Daily Traffic; DAC16 stands for Dense-graded Asphalt Cement with a nominal maximum aggregate size of $16 \mathrm{~mm}$; SMA11 stands for Stone Mastic Asphalt with a nominal maximum aggregate size of $11 \mathrm{~mm}$.

${ }^{\mathrm{a}}$ Heavy vehicle percentage $=($ number of heavy vehicles $/ \mathrm{AADT}) \times 100$.

${ }^{\mathrm{b}}$ Names of sites located in the city of Espoo, Finland: A (Konemiehentie), B (Mankkaanlaaksontie),C (Sinimaentie) and D (Keha I)

\section{Best cut plane for tyre/road contact}

The contact condition determines the average depth of the rubber penetrating into the substrate surface roughness. To properly correlate the friction coefficients with the roughness of the pavements, we need to identify this indentation depth on each pavement and later consider only this portion of the surface top topography that is actually contributing to the friction for analysis. In order to estimate the average indentation depth or the average interfacial separation $(\bar{u})$ between the rubber surface and the substrate mean plane [22], knowledge about the tyre rubber used in the friction device is important. However, for typical commercial friction testers that are designed for field experiments (as used here), the detailed characteristics of the measurement tyre are not known. Therefore, a realistic approach would be to choose the most relevant surface plane for top topography analysis of all pavements while the sensitivity of the correlation analysis to this surface cut is also investigated.

Hartikainen et al. [13] used a fixed depth of half the average $R_{q}$ of all the surfaces they studied for their top PSD calculations. From the correlation results, they deduced that using a fixed depth $\left(0.5 R_{q}\right)$ for top PSD calculation provides a better correlation with the friction results compared to a fixed surface area (a certain percentage of the top topography) approach. Here, we argue this and present support for the generic privilege of a fixed surface area method, i.e. choosing a certain percentage of the top topography rather than a specific depth for the top PSD calculations. We start with a discussion on the best cut plane for correlating road roughness with friction and give a rough estimation on the order of $\bar{u}$ between the tyre rubber and the pavements in this study. Persson [22] derived a relation between the average interfacial separation and the normal load for the contact of a rigid solid with a randomly rough surface. For the case of a self-affine fractal surface with a fractal dimension $D_{f} \leq 2.5$, he introduced a general form for the relation between the squeezing pressure and $\bar{u}[22]$ : 
where $\mathrm{p}$ is the squeezing pressure, $\epsilon \approx 0.7493, q_{0}$ is the roll-off wavevector of the surface (in case of asphalt pavements, $q_{0}$ is representative of the nominal aggregate size in a pavement roughness PSD), $E^{*}$ is the effective elastic modulus and $\gamma \approx 0.5$ [22,23]. Asphalt road surfaces have shown fractal behaviour with $D_{f} \approx 2.2$ [21]. Using Eq. 13, in Fig. 4, the relation between the nominal contact pressure and $\bar{u}$ is shown for a fractal surface with roughness parameters similar to the asphalt pavements under study. We now consider the worst case scenario for the order of $E^{*}$ in the calculations. Since the separation $\bar{u}$ is dominant by the long wavelength components of the roughness spectrum [22-24], for a typical summer tyre compound sliding (here, forward velocity $v \approx 14 \mathrm{~cm} / \mathrm{s}$ with a $20 \%$ slip ratio), the elastic modulus of the tyre rubber (measurement temperatures $-6 C^{\circ}$ to $21.5 C^{\circ}$ ) with perturbing frequencies $\omega \approx$ $q_{0} v \approx 10 \mathrm{~Hz}$ could not be lower than $10^{6.5} \mathrm{~Pa} \approx 3 \mathrm{MPa}$ for the highest temperature, where the strain softening of the rubber is also included. For lower temperatures, the viscoelastic master curve of the rubber shifts towards the shorter frequencies and $E^{*}$ would definitely be much larger than what has been estimated. Knowing that the nominal contact pressure is around $0.05 \mathrm{MPa}$ (portable friction tester equipped with small tyres), we see an interfacial separation $\frac{\bar{u}}{R_{q}}>1$ (Fig. 4, the grey region). Thus, here, the contact on each of the pavements must have happened above its $R_{q}$ height level. It is worth mentioning that calculating an exact depth for the penetration of rubber on each pavement is a challenging task. In particular, the whole tyre geometry as well as non-uniform pressure distribution in the contact patch affect the average interfacial separation between tyre and pavement. Eq. 13 applies to the contact of a rough rigid solid (here the pavement) with an elastic solid with flat surface such as flat tread blocks. With all these in mind, the rough estimation given in the manuscript considers the worst case scenario of the measurement conditions. Above all, the focus of the paper is not to present the best estimation method for rubber penetration onto each pavement, but to establish and introduce the correct methodology that one needs to adopt when correlating friction data with roughness, pointing out the limitations as well as practical application of top PSD method along with the future challenges faced during these types of correlation analysis.

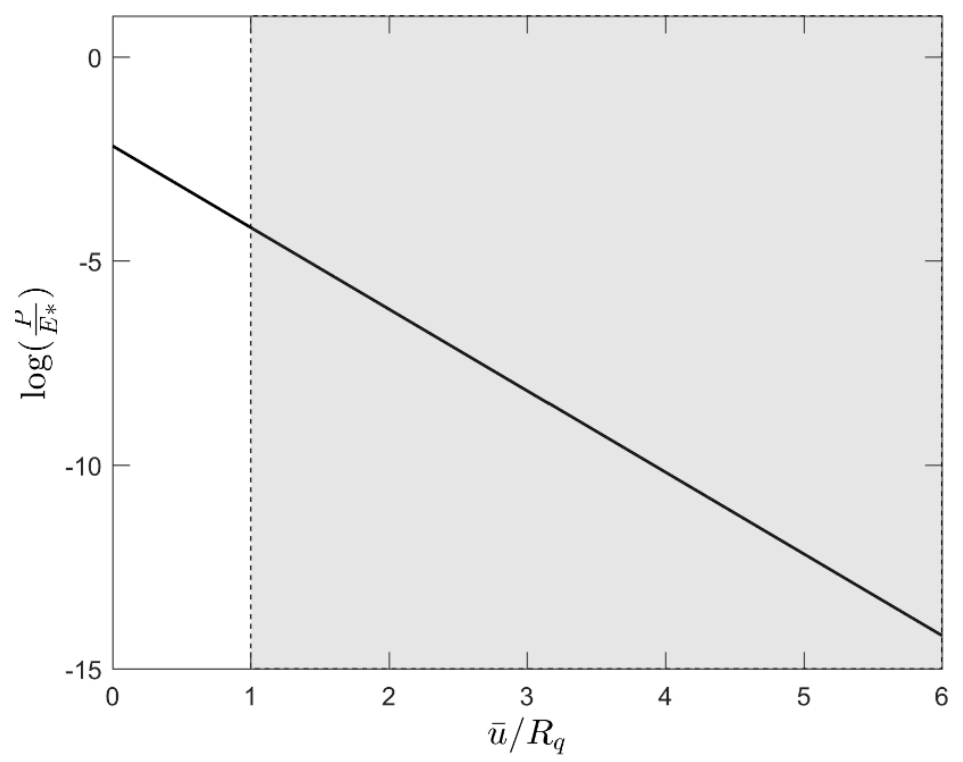

Fig. 4. The relation between the (natural) logarithm of contact pressure to the average interfacial separation $\bar{u}$ for the contact between a rigid solid with a randomly rough fractal surface with $\mathrm{H}=0.8, \mathrm{q}_{0}=500$ and $\mathrm{R}_{\mathrm{q}}=0.6 \mathrm{~mm}$.

Now, for a randomly rough surface of Gaussian height distribution, we know that about $68 \%$ of the data are within one standard deviation $\sigma\left(=R_{q}\right.$ for zero mean) away from the mean. Seeing that $\bar{u}>R_{q}$, and $50 \%$ of the data builds 
the top topography, the contact with such a surface must have happened in the top $16 \%$ of the data. In reality, asphalt pavements are skewed, of which the main consequences are twofold: first, they increase the $R_{q}$ of the pavement, while this rise does not influence the contact mechanics and second, they relocate the mode of the height distribution (the most frequent value in height distribution) and the mode no longer occurs at the mean plane of the surface topography. For the pavements under study, the mode and median of the height distribution were so close to each other that it is suggested that the best method to exclude the impact of the bottom profile would be to calculate the roughness indicators for the top $50 \%$ of the surface. In that event, the relation $\bar{u}>R_{q}$ holds when $R_{q}$ is calculated only for the top $50 \%$ of the data and the contact on the four pavements must have happened on the top $16 \%$ of the surface topographies.

Even though an estimation of the portion of the surface that the rubber penetrates is given above, the question still remains of whether to cut the topography at a fixed depth or a fixed surface ratio. To deal with this issue, we first discuss the relation between depth and area ratio for asphalt pavements. The best method to visualize this relation is through the use of the Abbott-Firestone curve or the Bearing Area Curve (BAC) of the surface topography. An illustration of the BAC for a randomly rough fractal surface is presented in Fig. 5(a). This curve is formed by cutting the surface topography at different depths from the top of the asperities to the bottom of the deep valleys and then calculating the percentage of the surface area that intersects with the cut plane. Ech et al. [25] were the first to introduce the application of the BAC to evaluate the evolution of the macrotexture of pavements and here we scrutinize this application in the evaluation of tyre/road friction. Literally, BAC is the cumulative probability density function of the surface height profile; thus, the main parameter influencing its shape is the standard deviation $\sigma$ of the height distribution. As shown in Fig. 5(b) for fractal topographies, reducing the aggregate size, i.e. increasing the roll-off wavevector $q_{0}$, has no influence on BAC unless $\sigma$, which is an indicator of the full surface topography, differs in the pavements. Therefore, BAC, similar to many other roughness indicators, is incapable of correlating the evolution of top surface roughness to friction data. Here, we restate the complexity of this system, seeing that none of the typical roughness characterization techniques works well when it comes to tyre/road contact phenomena. Nevertheless, BAC is employed in the present study to indicate the relation between the depth and the area percentage of the pavement topographies.
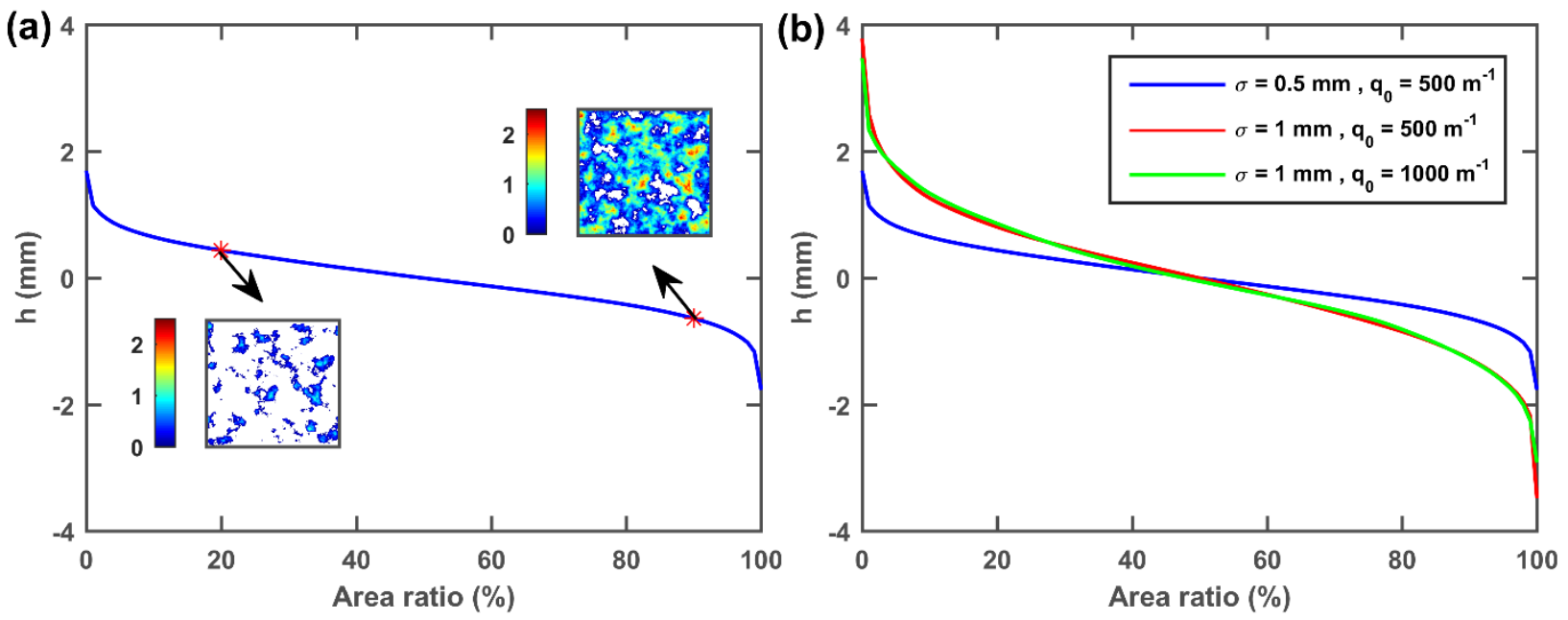

Fig. 5. The relation between depth and surface area using Bearing Area Curve BAC: (a) Typical BAC for a randomly rough fractal surface. (b) On the effect of $R_{q}$ and $q_{0}$ on the behaviour of BAC.

Looking at Fig. 6(a), where the BAC for a typical pavement topography is exemplified, we observe that the BAC divides the surface profile into three distinct zones: I. the peak zone where the top of the stone asperities are located, alongside the fact that this is the surface portion of the profile that we are interested in; II. a linear zone, where the bodies of the stones are covered; III. another crucial section of the surface topography, specified as valleys that hold rainwater on the pavement. A comparison of the BAC of the four pavements that were studied in Fig. 6(b) finally 
helps us to conclude that if we assign a common depth for the top PSD analysis, this height level may hold $20 \%$ of the top surface profile in one pavement, while including over 50\% of the surface area in the other asphalt surface. Therefore, it only makes sense to choose a unique depth for analysis if the pavements under study share a similar $R_{q}$, which is typically not the case. It is worth mentioning that the difference between the skewness of the surfaces can clearly be observed from the BACs, meaning that the water-handling capacity of the pavements is different. This may influence the depth of the water layer on the surfaces in the course of wet friction experiments and must be accounted for during data analysis. In the present study, it is not an issue as the friction experiments were carried out in dry conditions.
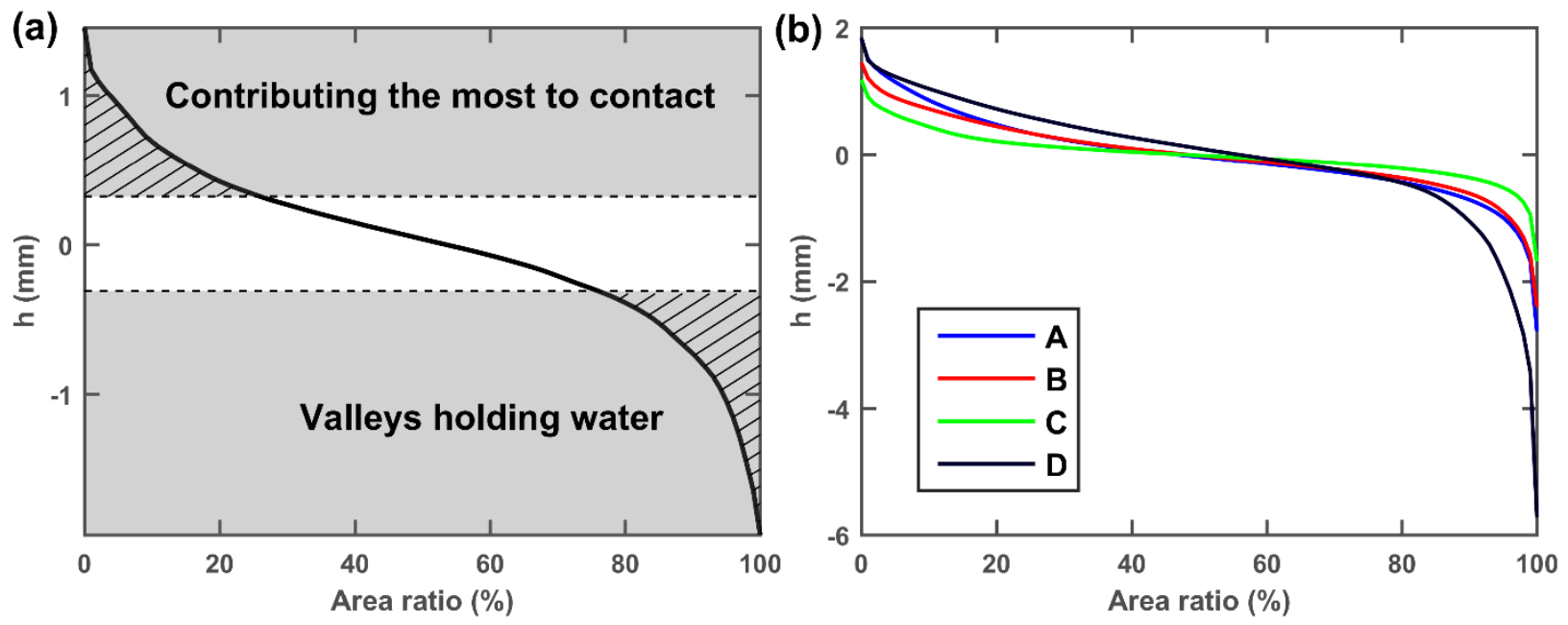

Fig. 6. Bearing area curve BAC of surface topography. (a) Typical BAC of a pavement. (b) Comparing the BACs of the pavements under study on one of the test dates.

In general, we are interested in seeing the influence of the percentage of data (if any) on the calculated top PSD at different wavelengths $\lambda$. This is illustrated in Fig. 7 for the pavements under study. As follows from the figure, there is a major difference between the full PSD (100\% area ratio) and the PSD calculated at the top, say, 20\% surface profile, which is prominent for pavement $\mathrm{D}$, with the highest skewness, as already shown in Fig. 6 (b). For $\lambda>1 \mathrm{~mm}$, for instance considering $\lambda=6 \mathrm{~mm}$, the variation in PSD as a function of the area ratio is usually more evident compared to shorter wavelengths, since with the increase in the surface ratio involved, the longer wavelengths gradually appear in the calculations, which are in general sensitive to the pavement profile. If road surfaces behave similarly to a fractal surface, then to evaluate the short-scale roughness, no best cut plane exists (see Fig. 2(b)). This statement is almost true for the shortest wavelength in the graph, $\lambda=200 \mu \mathrm{m}$ in an area ratio $\leq 80 \%$. Typically, for area ratios below $20 \%$, which is the area we are mostly interested in, the variation in the power spectrum as a function of $\lambda$ increases with a rise in $\lambda$. This can later affect the correlation analysis of roughness PSD with friction and is discussed in the next section during the correlation analysis between the friction results and the roughness at different length scales. 

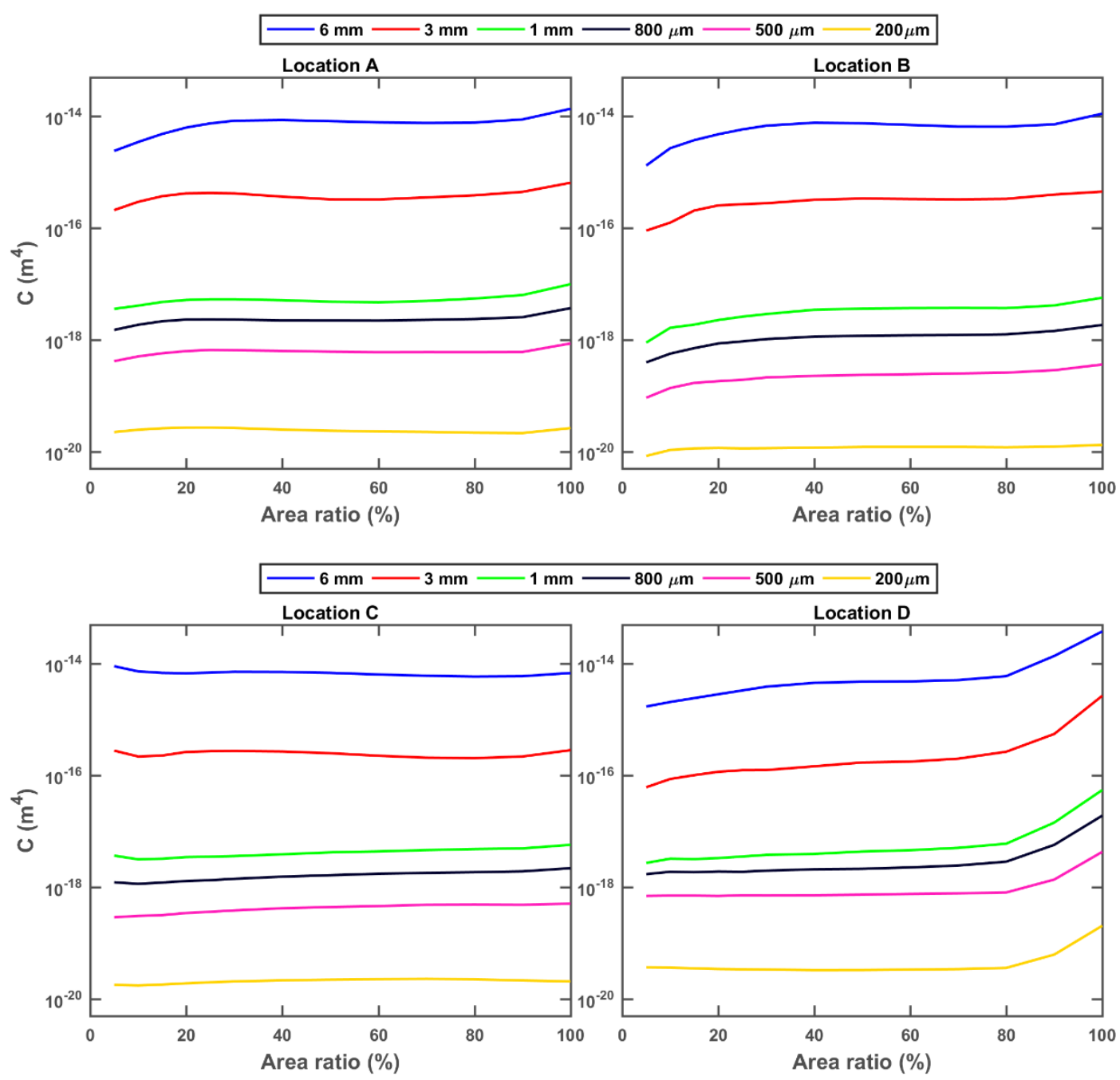

Fig. 7. Effect of percentage of data on the PSD of surface profiles at different wavelengths for the four pavements on one of the test dates.

\section{Relation between top PSD and friction}

Rubber friction on road surfaces is a complex problem with different contributory factors [26]. If we consider the contribution of hysteresis to be dominant in tyre/road interaction at the high velocities relevant to this application, according to the theory developed by Persson [2]:

$\mu=\frac{1}{2} \int_{q_{L}}^{q_{1}} d q q^{3} C(q) P(q) \int_{0}^{2 \pi} d \phi \operatorname{Im} \frac{E(q v \cos \phi)}{\left(1-v^{2}\right) \sigma_{0}}$

where $C(q)$ is the surface roughness power spectrum PSD and $P(q)$ is the area of real contact with the nominal contact area $\left(\frac{A}{A_{0}}\right)$, which itself depends on $C(q)$. According to these relations, it is obvious that the relation between friction and roughness, even if the roughness is treated at many scales by means of the surface roughness power spectrum, is not so simple to achieve. In principle, all frequencies are a priori equally important for rubber friction [2]. Although the short-scale roughness usually makes the dominant contribution to the area of real contact [27] and viscoelastic deformations, the contact stiffness which defines the depth of the rubber penetration is dominant by the longwavelength components of the road roughness profile $[23,24]$. 
The purpose of mentioning the complexity of the friction/roughness relation was to remain cautious when interpreting the results of the correlation analysis. Now, according to the earlier estimation of the depth of penetration of the rubber, the top 20\% of the surface profile is first chosen for the top PSD calculations. For each test date, the correlation between the friction coefficients of the four pavements with the PSD at every $\lambda$ is studied. In this fashion, the influence of the temperature, which, on the evidence of our earlier study [15], is the most influential parameter in friction/roughness correlation, is excluded. The results of the analysis of the correlation between the friction coefficients with roughness in the two limited cases of $\lambda=200 \mu \mathrm{m}$ and $\lambda=500 \mu \mathrm{m}$ is presented in Fig. 8 for the seven test dates that were studied. As follows from the figure, other than April, a high correlation, even around 1, is observed between the friction and short-scale roughness. Although the measurements were carried out in dry conditions, because of the freezing weather conditions on some dates, there is a possibility of ice particles appearing on the test track of a pavement.

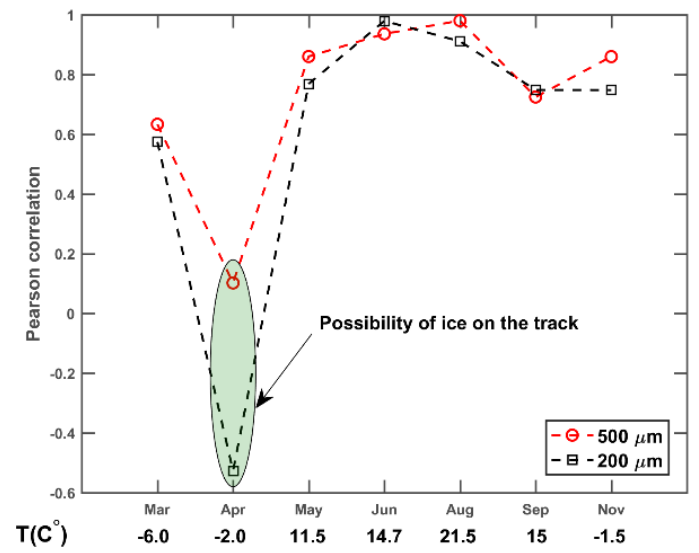

Fig. 8. Correlation between friction coefficients and roughness at $\lambda=200 \mu \mathrm{m}$ and $\lambda=500 \mu \mathrm{m}$ for different test dates.

Therefore, excluding the April data sets, similar results to the previous graph are introduced in Fig. 9 (a), but this time including all the roughness length scales relevant to this application. Since it is impractical to enter very long wavelengths in the correlation analysis because of the filtering nature of the top PSD calculations, only the results for $\lambda<6.3 \mathrm{~mm}$ are shown. The results of $\lambda<120 \mu \mathrm{m}$ are also avoided because of the inaccuracies of the optical profiler at these high frequencies. The solid line in the figure is the average of the correlation coefficients for the six dates, while the grey regions show the upper and lower boundaries. There are two main facts that can be derived from the figure. First, there is considerable variation in the results, especially for the longer wavelengths. If we again try to exclude data sets where the ambient temperature was below zero degrees $\left(\mathrm{C}^{\circ}\right)$, we come to Fig. 9 (b), where almost equal variations in the correlation are observed among different wavelengths. Although the considerable variations in the results could be attributed to the presence of ice particles at sub-zero ambient temperatures, because of the complexity of rubber friction and its strong dependence on temperature (and sliding velocities, which are not a constant value in a rolling tyre, as is the case here), no final conclusion can be drawn at the moment.

Second, the highest correlation with an average around 0.8 is only observed for $\lambda \leq 1 \mathrm{~mm}$. Admitting the importance of this finding on the correlation between the top $20 \%$ short-scale roughness and friction, we declare that the opposite conclusion for $\lambda>1 \mathrm{~mm}$ should not be concluded from the results. The fact that the average correlation drops to zero (see Fig. 9(b)) as the wavelength increases from $1 \mathrm{~mm}$ to $6 \mathrm{~mm}$ does not mean that the contribution of longer wavelengths is insignificant for rubber friction. Hartikainen et al. [13], observing similar behaviour to us, concluded that the shortest wavelengths contribute the most to wet rubber friction. While this statement is generally true in terms of the contribution of hysteresis to the friction [28], we argue that the observation on longer wavelengths probably originates to a great extent from the error introduced by the earlier assumption that was made in choosing a common depth (Hartikainen et al. [13]) or a common area ratio (the present work) for calculating the top PSD. Even though a common area ratio (top 16\%) approach was shown to be a better method in estimating the portion of the top pavement topography involved in tyre/road interaction, still, the exact depth of the penetration of the rubber must have been different on different pavements and the rubber sees the longer wavelengths differently on each pavement. 
Therefore, the observation in Fig. 9 should be linked to the information in Fig. 7, where we concluded that even for an area ratio $\leq 20 \%$, the top PSD changes as a function of area ratio, in which the variations are more prominent for a longer $\lambda$. Without exact knowledge of the penetration depth of the rubber on each pavement, which is a challenging task, the correlation analysis of friction and roughness using the top PSD as the roughness indictor is limited to meaningful correlations only with the short-scale roughness (here the microroughness). In order to see the robustness of this approach, in Fig. 10, we present the results of frequency-wise correlation analysis between all the friction measurements and all the roughness measurements. As already expected, the correlation drops when all the experimental data are considered together. This is in accordance to our previous study [15] where a high correlation was only noticed for the correlation between friction and temperature. Here, the measurement temperatures ranged from $-6 C^{\circ}$ to $21.5 C^{\circ}$ which significantly affect the viscoelastic response of the tyre rubber to the perturbing frequencies coming from the road roughness.

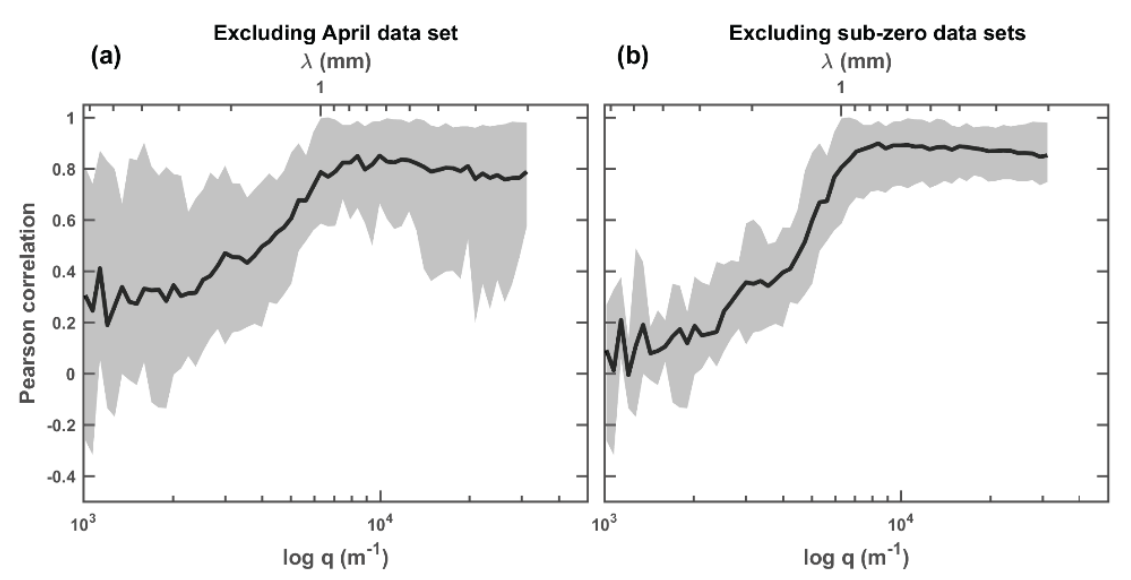

Fig. 9. Correlation between friction coefficients and roughness at all length scales. The solid lines are the average correlation of different dates, with grey regions showing the upper and lower boundaries of correlation.

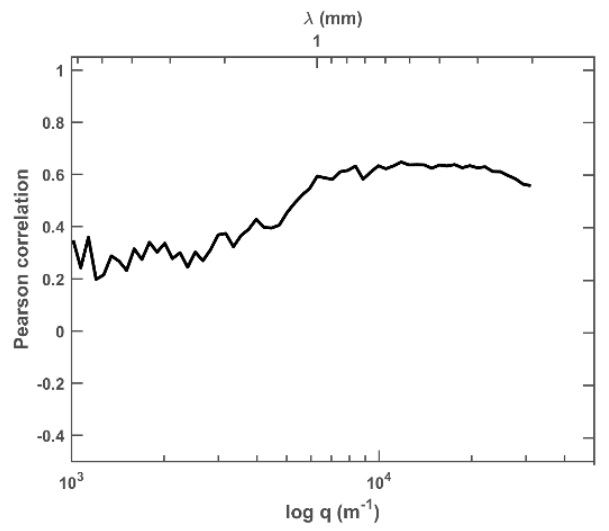

Fig. 10. Correlation between all the friction data and all roughness data at different length scales.

Last, we briefly address the results of the correlation analysis for other area ratios than the top 20\%. Although, on the basis of the early estimation of the penetration depth, the top $20 \%$ was selected as a good approximation of the relevant portion of the top topography involved in the contact, it is worthwhile to take a look at the correlation results shown in Fig. 11 for 20\%, 60\% and 100\% surface topographies. As previously observed in Fig. 7, for shorter $\lambda$ and area ratios below $80 \%$, the roughness does not change considerably with a change in the area ratio. Thus, it is trivial that for the shorter length scales of the 60\% area ratio graph in Fig. 11, similar correlation levels to the $20 \%$ case can be noticed. 
For $\lambda>1 \mathrm{~mm}$, the discussion presented in the previous paragraph is valid when $20 \%$ and $60 \%$ correlation patterns are compared. Finally, in the case of full topography (100\%), we can see that the correlation in short-scale roughness drops, as we also expected from the beginning that any indicators of the roughness derived from the full surface profile could not relate the roughness to the friction well. For longer wavelengths, a weak correlation with friction is seen for the PSD calculated at 100\% area ratio. However, based on the results presented in Fig.6 (b), the pavements (especially pavement D) have skewed topographies, or in other words, the bottom profiles have deep valleys which are not actually contributing to dry friction. This skewness affects the calculated PSD for $100 \%$ area ratio, as already shown in Fig.7 where the PSD outcomes rise for the $100 \%$ area ratio, and the magnitude is largest for the most skewed pavement. In short, if the skewness of a pavement is zero, then probably the calculated PSD based on the 100\% area ratio shows some correlation (if exists) between long wavelengths and friction. But, for correlation analysis in the case of skewed pavements (here), the PSD calculated based on $100 \%$ area ratio is affected by the bottom profile which has no contribution to friction. Therefore, although a weak correlation of friction with long wavelengths is noticed in Fig.11, we would rather not make a conclusion formed on this observation, as we don't have a clear support for that.

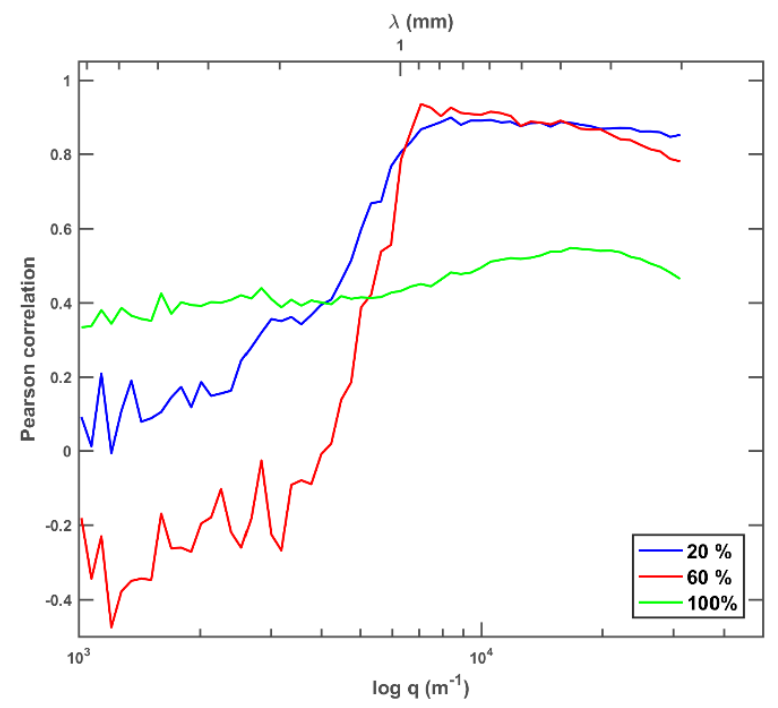

Fig. 11. The influence of the top PSD area ratio on the correlation results.

\section{Conclusion}

We described the most approperiate method for calculating the top surface roughness power spectrum. The realization given by the top PSD about the surface roughness components and the asperity distributions was illustrated for different top portions of the surface topography. It was shown that the top PSD calculated at any portion of the top surface profile except the top 50\% leads to false conclusions about the actual asperity distributions. However, such a top PSD at any top portion of the surface profile could be utilized for characterization of the surface roughness at different length scales.

We addressed the difficulty that is faced when finding the correlation between roughness and friction for field experiments. Such a correlation between the friction and the roughness at different length scales (from macro- to micro-scale) requires exact knowledge of the depth of the penetration of the rubber into the asperities in the pavement. 
This depth was roughly estimated to be in the top $16 \%$ of the surface topographies of the pavements in the present study. The results showed that the area ratio of the surface topography used for the top PSD calculations generally influences the resulting roughness power spectra of road pavements.

Here, a high correlation with an average around 0.8 was found with the friction and the top $20 \%$ of PSDs, but only at short-scale surface roughness $\lambda \leq 1 \mathrm{~mm}$. It was manifested that both defining a common depth and a common surface area ratio during the top PSD calculations impose an intrinsic error on the later correlation results of friction with long $\lambda$, since the rubber sees the long wavelengths differently on different pavements. In other words, it is only through the estimation of the depth of the penetration of the rubber into each pavement and later calculating the top PSD at this certain depth that a reliable correlation can be achieved between friction and all the roughness length scales relevant to the rubber friction. On the basis of the findings and the procedure that was developed, the top PSD is introduced as being by far the most relevant method for correlating pavement roughness at short length scales to the rubber friction.

\section{Acknowledgements}

We would like to thank Antti Kuosmanen for his contribution to the measurements. This work was funded by the Academy of Finland (grant numbers 138272 and 274516).

\section{References}

[1] Klüppel M, Heinrich G. Rubber Friction on Self-Affine Road Tracks. Rubber Chem Technol 2000;73:578606. doi:10.5254/1.3547607.

[2] Persson BNJ. Theory of rubber friction and contact mechanics. J Chem Phys 2001;115:3840-61. doi:10.1063/1.1388626.

[3] Persson BNJ. Rubber friction: role of the flash temperature. J Phys Condens Matter 2006;18:7789-823. doi:10.1088/0953-8984/18/32/025.

[4] Persson BNJ. Role of Frictional Heating in Rubber Friction. Tribol Lett 2014;56:77-92. doi:10.1007/s11249-014-0386-0.

[5] Yang C, Persson BNJ. Molecular dynamics study of contact mechanics: Contact area and interfacial separation from small to full contact. Phys Rev Lett 2008;100. doi:10.1103/PhysRevLett.100.024303.

[6] Scaraggi M, Persson BNJ. General contact mechanics theory for randomly rough surfaces with application to rubber friction. J Chem Phys 2015;143. doi:10.1063/1.4936558.

[7] Carbone G, Lorenz B, Persson BNJ, Wohlers A. Contact mechanics and rubber friction for randomly rough surfaces with anisotropic statistical properties. Eur Phys J E 2009;29:275-84. doi:10.1140/epje/i200910484-8.

[8] Chen XH, Wang DW. Fractal and spectral analysis of aggregate surface profile in polishing process. Wear 2011;271:2746-50. doi:10.1016/j.wear.2011.05.024.

[9] Do MT, Cerezo V, Beautru Y, Kane M. Modeling of the connection road surface microtexture/water depth/friction. Wear 2013;302:1426-35. doi:10.1016/j.wear.2013.01.031.

[10] Do MT, Tang Z, Kane M, de Larrard F. Evolution of road-surface skid-resistance and texture due to polishing. Wear 2009;266:574-7. doi:10.1016/j.wear.2008.04.060.

[11] Do MT, Tang Z, Kane M, de Larrard F. Pavement polishing-Development of a dedicated laboratory test and its correlation with road results. Wear 2007;263:36-42. doi:10.1016/j.wear.2006.12.086.

[12] Dunford AM, Parry AR, Shipway PH, Viner HE. Three-dimensional characterisation of surface texture for road stones undergoing simulated traffic wear. Wear 2012;292-293:188-96. 
doi:10.1016/j.wear.2012.05.010.

[13] Hartikainen L, Petry F, Westermann S. Frequency-wise correlation of the power spectral density of asphalt surface roughness and tire wet friction. Wear 2014;317:111-9. doi:10.1016/j.wear.2014.05.017.

[14] Kuosmanen A, Pellinen T, Hartikainen L, Petry F, Westermann S. Durable laboratory rubber friction test countersurfaces that replicate the roughness of asphalt pavements. Wear 2014;321:38-45.

doi:10.1016/j.wear.2014.09.011.

[15] Mahboob Kanafi M, Kuosmanen A, Pellinen TK, Tuononen AJ. Macro- and micro-texture evolution of road pavements and correlation with friction. Int J Pavement Eng 2015;16:168-79.

doi:10.1080/10298436.2014.937715.

[16] Torbruegge S, Wies B. Characterization of pavement texture by means of height difference correlation and relation to wet skid resistance. J Traffic Transp Eng (English Ed 2015;2:59-67.

doi:10.1016/j.jtte.2015.02.001.

[17] Ueckermann A, Wang D, Oeser M, Steinauer B. A contribution to non-contact skid resistance measurement. Int J Pavement Eng 2015;16:646-59. doi:10.1080/10298436.2014.943216.

[18] Wang D, Chen X, Oeser M, Stanjek H, Steinauer B. Study of micro-texture and skid resistance change of granite slabs during the polishing with the Aachen Polishing Machine. Wear 2014;318:1-11. doi:10.1016/j.wear.2014.06.005.

[19] Villani MM, Scarpas A, de Bondt A, Khedoe R, Artamendi I. Application of fractal analysis for measuring the effects of rubber polishing on the friction of asphalt concrete mixtures. Wear 2014;320:179-88. doi:10.1016/j.wear.2014.08.013.

[20] Kanafi MM, Tuononen AJ. Application of three-dimensional printing to pavement texture effects on rubber friction. Road Mater Pavement Des 2016;629:1-17. doi:10.1080/14680629.2016.1194883.

[21] Persson BNJ, Albohr O, Tartaglino U, Volokitin AI, Tosatti E. On the nature of surface roughness with application to contact mechanics, sealing, rubber friction and adhesion. J Phys Condens Matter 2005;17:R162. doi:10.1088/0953-8984/17/1/R01.

[22] Persson BNJ. Relation between interfacial separation and load: A general theory of contact mechanics. Phys Rev Lett 2007;99:1-4. doi:10.1103/PhysRevLett.99.125502.

[23] Wang D, Ueckermann a., Schacht a., Oeser M, Steinauer B, Persson BNJ. Tire-Road Contact Stiffness. Tribol Lett 2014;56:397-402. doi:10.1007/s11249-014-0417-x.

[24] Popov VL, Dimaki A, Psakhie S, Popov M. On the role of scales in contact mechanics and friction between elastomers and randomly rough self-affine surfaces. Sci Rep 2015;5:11139. doi:10.1038/srep11139.

[25] Ech M, Morel S, Yotte S, Breysse D, Pouteau B. An Original Evaluation of the Wearing Course Macrotexture Evolution using the Abbot Curve. Road Mater Pavement Des 2009;10:471-94. doi:10.3166/RMPD.10.471-494.

[26] Lorenz B, Persson BNJ, Dieluweit S, Tada T. Rubber friction: Comparison of theory with experiment. Eur Phys J E 2011;34. doi:10.1140/epje/i2011-11129-1.

[27] Lorenz B, Persson BNJ, Fortunato G, Giustiniano M, Baldoni F. Rubber friction for tire tread compound on road surfaces. J Phys Condens Matter 2013;25:95007. doi:10.1088/0953-8984/25/9/095007.

[28] Lorenz B, Oh YR, Nam SK, Jeon SH, Persson BNJ. Rubber friction on road surfaces: Experiment and theory for low sliding speeds. J Chem Phys 2015;142:0-12. doi:10.1063/1.4919221. 
